\title{
Proceedings of the Society of British Neurological Surgeons
}

\author{
The 99th meeting of the Society of British Neurological Surgeons held in Sheffield, 24-25 September 1981
}

EXPERIENCES WITH CORDECTOMY FOR INTRACTABLE PAIN IN PARAPLEGIA

\section{A Jefferson (Sheffield)}

When post-traumatic paraplegia is accompanied by intractable pain, management is difficult and excision of the damaged cord may be helpful. The author reviewed 21 patients who had undergone this operation between 1967 and 1980. The mean follow-up was over $4 \frac{1}{2} \mathrm{yr}$. The average age of patients was $37 \mathrm{yr}$; all but two were male. Only one had a lesion above the D10 segment; in his case the length of cord lying beneath D3-4 vertebrae was excised. His pain was not influenced. The favourable results had followed treatment for lesions in the low thoracic or upper lumbar regions. The number operated represented about $6 \%$ of all admissions with thoracolumbar injuries.

The pains had all lain within the anaesthetic area and were usually approximately symmetrical. They were frequently episodic and sometimes had a shooting quality which might be so pronounced that the patient cringed momentarily, as does a sufferer from trigeminal neuralgia. Burning pains had not usually occurred. In the successful cases the pain had been felt predominantly but not absolutely exclusively on the fronts of the legs, either as a blob around the knees or as a patch in the mid-thigh. Less consistent success had followed cordectomy when the pain was located more diffusely throughout the thighs and lower legs. Cordectomy had been ineffective for pains in lower abdomen, sacrum, or rectum.

The cord was usually excised from the lower border of D10 and D11 vertebra down to the conus. Because of the severity of the initial cord trauma, cordectomy had not apparently damaged bladder function. No patient had died in the postoperative period; moreover, apart from sometimes raising the neurological level by up to approximately one segment no significant postoperative morbidity had occurred.
TRAUMATIC INTRACRANIAL HAEMATOMA: ARE WE DOING BETTER?

G Teasdale, S Galbraith, L Murray, P Ward, D Gentleman, M McKean (Glasgow)

Evidence from several sources shows that many head-injured patients die or become disabled from brain damage that results from unduly delayed treatment of a traumatic intracranial haematoma. The authors suggested that this was the result of a traditional policy of transferring patients to a neurosurgical unit only after the need for operation had been made obvious by the onset of deteriorating responsiveness. They reported 3 years experience with a more liberal admission policy, aimed at early detection of a traumatic haematoma.

Compared with the 4 previous years, there had been a doubling in the number of head-injured patients admitted to the neurosurgical unit and an increase from 76 to 126 per year in patients with a traumatic haematoma requiring evacuation. The patients undergoing operation in the two periods were similar with respect to the type of haematoma, age and evidence of primary brain damage. In the early period, $46 \%$ of patients who had been talking after injury later deteriorated into coma before operation but this proportion was reduced to $24 \%$ after the change in admission policy. Along with this, there had been reduction in overall mortality from $38 \%$ to $29 \%$.

The authors speculated that some of the persisting mortality was due to the admission to the neurosurgical unit of patients who would previously have died in a general hospital and drew attention to the complex consequences of changes in admission policy. Nevertheless, they believed that the admission of even more head-injured patients, sooner, to a neurosurgical unit can result in improved outcomes.

CEREBRAL AVM IN ASSOCIATION WITH CEREBRAL ANEURYSM RE Cheeks, RH Shephard (Derby)
A patient who has both an arteriovenous malformation and an associated arterial aneurysm poses a problem: should both lesions or only one be dealt with, and if the latter, which one? The authors had encountered this combination in eight of 135 patients with a cerebral arteriovenous malformation over a 23 year period. The eight patients were normotensive, averaged 41 yrs of age, and consisted of equal numbers of male and female. A few patients had multiple aneurysms. In six patients the aneurysm was located on the vessel feeding the AVM.

The aim at operation had been to deal with the lesion that had caused the presenting feature. In three patients this has been due to aneurysm rupture and these have been managed by clipping of the aneurysm and, if feasible, excision of the AVM. This was not possible in one patient and the AVM bled later, but not fatally. Three patients presented with features of arteriovenous malformation and this had been excised, with clipping of the aneurysm performed if practicable. This had not been possible in one patient who died later from rupture of the aneurysm. A further patient, whose basilar aneurysm had caused hydrocephalus, died due to rupture of the aneurysm following an operation for CSF diversion. The final patient, with a thalamic arteriovenous malformation, had undergone only an exploratory operation.

The authors suggested that when dealing with this dangerous combination of lesions, the presenting lesion should be treated on its merits but that the aneurysm be regarded as a greater menace. Their review of the available literature produced further support for this view.

THE INFLUENCE OF CAROTID ARTERY FLOW ON THE PLANNING OF CEREBRAL REVASCULARISATION PROCEDURES

CM Bannister (Manchester)

The author presented three patients from more than 50 who had undergone an extracranial-intracranial anastomoses, in order to stress the importance of com- 
prehensive angiographic assessment in the planning of revascularisation. In two patients carotid angiography had apparently shown occlusions of the internal carotid artery above a tight stenosis at the origin. In one patient there had even been no back-flow during a disobliterative endarterectomy. Nevertheless, in both patients check angiography after anastomoses had shown that the internal carotid was patent and perfusing the intracranial circulation. In one patient there was even reflux from intracranial to extracranial vessels through the anastomosis. The third patient had occlusions of common and internal carotid arteries; although occlusion of the external carotid artery would have been anticipated, the vessel on one side was filling faintly from collaterals. This permitted an effective revascularisation, employing a vein graft from subclavian to external carotid and a superficial temporal anastomosis to the intracranial circulation.

THE EFFECT OF FIBRIN(OGEN) DEGRADATION PRODUCTS ON HUMAN BASILAR ARTERY PREPARATIONS: POSSIBLE ROLE IN THE AETIOLOGY OF CEREBRAL ARTERIAL SPASM RH Lye, KS Paul, C Forster, ET Whalley, J Dutton (Manchester)

Fibrin(ogen) degradation products have been considered possible agents contributing to the aetiology of cerebral arterial spasm. The authors had investigated the effect of degradation products on the human basilar artery in vitro.

Aliquots of fibrin(ogen) degradation products produced by plasma/streptokinase incubates were taken 90 minutes, 48 hours, 1 week and 2 weeks after onset of incubation and separated into fractions of molecular weight $>100000 ;<100000$ $>10000$; and $<10000$ using ultrafiltration. Each fraction was tested for its contractile effect on spiral strip preparations of postmortem human basilar artery and also for its ability to enhance threshold responses of these arterial preparations to 5-hydroxy-tryptamine (5-HT), comparing activity with that of plasma controls. Contractile activity was confined to the mol wt $>100000$ fraction in the 90 minute samples but was also present in the $<100000>10000$ fractions after longer incubation. No contractile activity was found with any mol wt $<10000$ fractions. Enhancement of threshold 5-HT responses was limited to the mol wt $>100000$ and $<100000>10000$ fractions of the 90 minute incubate, but from 48 hours onwards the mol wt $<10000$ fractions also showed enhancing activity.

These findings are compatible with a proposed temporal sequence of breakdown of fibrin(ogen) into degradation fragments. Possibly the early products are involved in predominantly enhancing effects whereas later products have contractile activity. This would be consistent with the suggestion that cerebral arterial spasm is a biphasic event. It may be that degradation fragments contribute to cerebral arterial spasm by modulating or prolonging the effects of other factors.

THE SURGICAL TREATMENT OF CRANIOPHARYNGIOMA

L Symon, V Logue, J Jakubowski (London)

There is controversy about the relative merits of different forms of management of craniopharyngioma. The authors compared the outcomes of 101 patients whom they had treated by either an intracranial operation, with varying degrees of removal of tumour, or by aspirations. Thirteen patients were under 20 years old, three less than 10 years.

The results of three differing degrees of intracranial removal and of aspiration were presented. (1) radical excision was performed at the first operation in 19 patients and in seven other patients a radical operation was performed for a recurrence of a previously partially removed tumour. There were four operative deaths, one early death within 3 months and one unrelated death from coronary thrombosis seven years after operation. In 20 patients followed from 2 years to over 20 yrs there were no recurrences. Four patients had survived for more than $20 \mathrm{yr}, 10$ more than 5 , and 16 more than 3 yr. (2) a less radical operation was performed in 34 patients, a portion of the tumour being known to have been left behind. The operative mortality of this group was $3 \%$, with recurrence in 15 patients $(44 \%)$ : only 26 of these patients still survive. (3) partial removal was performed in 22 patients, with six operative deaths. There had been recurrence of symptoms sufficient to require further operation in 11 patients. At the time of review, there were 10 survivors, five of whom had required a further operation. Only five patients were alive without further surgery $(23 \%$ of the original group). (4) aspiration with biopsy and subsequent shunt was per- formed in 19 patients. There were two operative deaths, two early deaths, and six late deaths; one of the latter was unrelated to the disease. Sixteen patients in this group had undergone further operation because of recurrent symptoms. Nine patients were alive at review but only three of these had survived without further treatment.

The authors concluded that the best hope for freedom of recurrence was achieved by a maximal removal of accessible tumour at the first operation. This approach should be reconsidered in the light of improvements in management as a result of the adoption of the operating microscope and hormone therapy.

CENTRAL CONDUCTION TIME AS A FUNCTIONAL MONITOR IN BRAIN ISCHAEMIA

L Symon, J Cone, JR Hargadine, ADJ

Wang, A Watson (London)

Somatosensory evoked potentials from median nerve stimulation can be used to assess conduction through the central nervous system. The time measured is the difference between two negative peaks, one recorded over the second cervical spine, and the other over the contralateral somatosensory cortex. The time difference of the latency of these waves is termed the central conduction time.

In baboons with an acute middle cerebral artery occlusion, the authors found a hyperbolic relationship between: central conduction time and the absolute valueof cerebral blood flow once ischaemia had stabilised, and a significant correlation between central conduction time change and the decrease in flow in the acute phase. In the stable phase, rCBF depression below $15 \mathrm{ml} / 100 \mathrm{gm} / \mathrm{min}$ caused progressively larger changes in central conduction time until the cortex was electrically silent.

Clinical studies were carried out before and after operation in patients with subarachnoid haemorrhage. In 13 patients whose arteriogram showed no intracranial aneurysm the average central conduction time was $5.4 \pm 0.4 \mathrm{~ms}$; the significant prolongation was therefore taken as $6.4 \mathrm{~ms}$ (normal \pm+2 standard deviation). In 44 patients with a ruptured aneurysm, the central conduction time before operation was related to the clinical grade. In 22 grade II patients it was $5.77 \pm 0.3 \mathrm{~ms}$. In 14 patients in grade III it was $5.94 \pm 0.66 \mathrm{~ms}$; and in eight patients in grade IV the central conduction time recorded from the hemisphere bearing the aneurysm or related to clinical 
features was $6 \cdot 11 \pm 0.62$ ms. After operation, the patients whose two-month outcome was poor (two deaths and eight patients with appreciable neurological deficits) showed significant prolongations of the central conduction time in the affected (aneurysm bearing) hemisphere from the first day. Patients with a good two-month outcome had a central conduction time within the normal range. Interhemispheric differences were studied and an increasing difference between the central conduction time on the two sides correlated with poor outcome. In individual patients, lengthening of conduction time appeared as a premonitor of clinical symptoms whereas a shortening of central conduction time could result from therapy such as induced hypertension. The authors commended the technique as having diagnostic, prognostic, and management value.

EFFECT OF SURGICAL MANIPULATION ON GLOBAL CEREBRAL BLOOD FLOW DURING CEREBRAL ANEURYSM SURGERY

JD Pickard, AHJ Lovick, BA Goddard (Southampton)

The intraoperative measurement of global cerebral blood flow (intravenous ${ }^{133}$ Xenon) has revealed that patients with defective autoregulation of cerebral blood flow are at much greater risk of developing late cerebral ischaemia after aneurysm surgery. The original method analysed the data off-line and was limited to six runs/ patient. The authors described a new method that provided the cerebral blood flow result within $6 \mathrm{~min}$ of the ${ }^{133} \mathrm{Xe}$ injection and enabled 15-20 runs/ patient. They used a modified Apple II microprocessor, teletype interface and nucleonics built into a purpose-built trolley for theatre use. The envelope of the expired air curve was defined using a joystick control.

In patients undergoing craniotomy for aneurysmal subarachnoid haemorrhage, skin incision provoked a $47 \%$ increase in cerebral blood flow. The combination of dural opening, brain retraction and CSF aspiration had little immediate effect on global cerebral blood flow but local cerebal blood flow changes may have been masked. In nine patients, aneurysmal rupture provoked a significant, and sometimes dramatic, increase in global cerebral blood flow when arterial blood pressure was adequately maintained. This may correspond to the rapid brain engorgement that sometimes accompanies intraoperative aneurysmal rupture.

The authors plan to develop a preoperative test of cerebrovascular reactivity in the expectation that this will yield prognostic clues similar to those obtained by intraoperative measurements of autoregulatory capacity. In the confused patient, the use of a face-mask and stationary ${ }^{133} \mathrm{Xe}$ detectors may be avoided by using detectors on the chest to reflect alveolar ${ }^{133} \mathrm{Xe}$ clearance and miniature Cd-Te detectors to monitor ${ }^{133} \mathrm{Xe}$ clearance from the head.

INTRACEREBRAL HAEMORRHAGE IN NORTH EAST SCOTLAND

FP Nath, DM Nichols, CT Blaiklock, RJA Fraser (Aberdeen)

The authors had reviewed retrospectively 244 patients who had suffered an intracerebral haemorrhage in order to examine the relation between the number of clinical and computer tomographic features and outcome.

The patients have been investigated over a 5 year period. The haemorrhage was judged to be spontaneous in $62 \%$ of patients; due to trauma in $18 \%$ and aneurysm in $14 \%$. The overall mortality was $27 \%$. The indications for operation, which had been performed in $32 \%$ of patients, had included deteriorating responsiveness or a persistent failure to improve. Operation had been followed by a mortality of $18 \%$. The patients who required operation after the first $48 \mathrm{~h}$ had fared much better than those requiring an operation before this time. Other favourable features were good conscious level; small clot volume; midline shift less than $1 \mathrm{~cm}$; and a stable course within the first $48 \mathrm{~h}$. The aetiology of the bleed was not related to outcome but was related to the systolic blood pressure, which was lower in those with a proven cause for bleeding.

The indications for operation were discussed; it was pointed out that, although outcome was less favourable in those operated on early, more than one-third of even these patients made acceptable recoveries.

RESULTS AND FOLLOW-UP OF 181 CASES OF ONLAY TITANIUM CRANIOPLASTY

TF Fannin, MJG Hawe, DS Gordon, GAS Blair (Belfast)

The stimulus to improve cranioplasty technique has usually resulted from the effects of head injuries sustained during war. Since the onset of civil disturbances in Northern Ireland in 1969 many of the penetrating head injuries have resulted from high velocity gunshot wounds, which leave in their wake considerable bony damage. This has resulted in the development of the onlay technique of titanium cranioplasty and a review of a large series was presented.

Of the 181 cases in the study, 138 $(76 \%)$ were male and $43(24 \%)$ female; the ages ranged from 10 months to 67 years. The causes of the skull defects include road traffic accidents $(21 \%)$, other accidents $(30 \%)$, "normal" neurosurgical disorders $(27 \%)$ and civil disturbance $(22 \%)$. The size of the largest plate in this series was $220 \mathrm{~cm}^{2}$. The indications for cranioplasty were (a) cosmetic and (b) protective, whether physical or psychological, and in 14 cases cranioplasty was performed at the time of primary injury or surgery. In $47 \%$ the interval from injury or primary surgery to cranioplasty was less than 26 weeks.

The results were extremely good with respect to both cover and contour. Infection occurred in three cases, $1.6 \%$. Plates were removed for reasons other than infection, including subsequent injury and the need for re-exploration. The mortality was zero and the morbidity low.

NEUROSURGICAL PROBLEMS IN CRANIOFACIAL SURGERY

AD Hockley, $\mathrm{H}$ Goldin, $\mathrm{M}$ Wake (Birmingham)

There is increasing interest and specialisation in the surgery of cranio-facial disorders. The authors had treated 19 patients in the two previous years and presented their indications, operative procedures, results, and complications. Thirteen patients had congenital anomalies (mostly Crouzon's or Apert's syndromes); two patients had extensive basal tumours, three had sustained a head injury; one patient had proptosis consequent on a previous orbital decompression.

The authors had found, in addition to conventional radiography of the face and cranial vault in two plains, that $C T$ scanning was more helpful than conventional skull tomography. The only death had occurred in a patient with an osteogenic sarcoma of the orbit which had recurred eight months after surgery. The most frequent complication was a persisting cerebro-spinal fluid leak, either through the wound or into the orbit. In three patients a leak had been controlled by a shunt. The authors emphasised the need for careful pre-operative assess- 
ment and for post-operative management involving the co-operation with several disciplines.

\section{THE HYDROCEPHALIC MUTANT MOUSE}

J Punt, J Pickard, R Weller, JM Mitchell, C Mather, S Perry, R Hunt (Southampton) The mechanisms by which hydrocephalus produces neurological dysfunction are poorly understood. The authors had developed a new inbred strain of hydrocephalic mouse and presented its characteristics. Tests of neurological development showed that the animals did acquire a gait disturbance, affecting the hind limbs. Intraventricular and intra-cisternal injections studies showed that the hydrocephalus was of a communicating type. The animals were thus similar to previously described strains of hydrocephalic mouse. Radiographic studies, performed following silver impregnation, showed that there were no concomitant skeletalabnormalities. Histological studies showed that there were degenerating axons in the brainstem of the affected animals but the relationship between these and the gait disturbance was unclear. Local cerebral glucose utilisation studies, using the autoradiographic technique of Sokoloff, had been performed in an effort to elucidate the mechanisms of gait disturbance. It was found that the technique could not be applied in its full quantitative form to a developing hydrocephalic mouse. Therefore, a rat model of hydrocephalus had been produced and studies of the effects of hydrocephalus on glucose utilisation in different neuronal systems are planned.

REGIONAL BLOOD FLOW AND OXYGEN METABOLISM IN CEREBRAL GLIOMA: APPLICATION OF OXYGEN-15 POSITRON EMISSION TOMOGRAPHY

DGT Thomas, M Ito, AA Lammerstma, R Rise, S Bernardi, RSJ Frackowiak, JO Heather, CG McKenzie, T Jones (London)

Little information exists about the physiology and metabolism of cerebral gliomas and adjacent surrounding brain. Histopathological studies of malignant gliomas commonly show areas of necrosis and it has been inferred that these tumours are hypoxic in vivo, both before surgery and subsequently during radiotherapy and chemotherapy. The presumed abnormalities in blood flow and oxygen metabolism of cerebral gliomas and altered function in normal adjacent brain may be relevant both to the difficulty in eradicating these tumours by radiation and chemotherapy following surgery and to the neurological dysfunction found in these patients.

The authors studied eight patients with cerebral glioma, using continuous inhalation of $\mathrm{C}^{15} \mathrm{O}_{2}$ and ${ }^{15} \mathrm{O}_{2}$ in combination with positron emission tomography in order to measure cerebral blood flow and oxygen metabolism and oxygen extraction ratio. The findings had been related to CT scan appearances. The results showed heterogeneous disturbances of cerebral blood flow and oxygen utilisation. Within the tumour oxygen extraction ratio was actually decreased, with an uncoupling between oxygen consumption and cerebral blood flow. In areas of peritumoral oedema there was a matched reduction in both blood flow and oxygen utilisation. Although the patients were conscious at the time of the study, there were also decreases in grey matter blood flow in areas remote from the tumour, even in the contralateral hemisphere.

While the studies were technically complex and the need for caution in interpreting the findings was noted, the results suggested that cerebral gliomas receive an oxygen supply that is sufficient for their metabolic needs, and may even be relatively overperfused.

THE CARBON DIOXIDE LASER IN NEUROSURGERY

RV Jeffreys (Liverpool)

The $\mathrm{CO}_{2}$ laser is applicable to neurosurgery in that its wavelength is efficiently absorbed by soft tissues, so that at differing intensities of power and of focus its properties can be varied from tissue cutting, blood vessel coagulation to vaporisation of tissues. It is important to expose target tissues to the least sufficient amount of energy and rapid intermittent exposure are preferable to long continuous exposures-in order to allow heat dissipation.

The author reviewed twelve months experience with a $\mathrm{CO}_{2}$ laser involving neurosurgical operations. The $\mathrm{CO}_{2}$ laser was used in conjunction with an operating microscope in 36 operations. These included giant pituitary tumours, III ventricular neoplasms, anterior fossa meningiomas and a variety of posterior fossa and spinal neoplasms. The author considered that there had been a significant reduction in blood loss and neurological morbidity though it was readily conceded that the number of operations was relatively small.

The particular advantages of the combination $\mathrm{CO}_{2}$ laser and microscope were felt to be precision (particularly using a micro-manipulator), reduction of mechanical trauma (less instrumentation being necessary), good haemostasis (of small vessels up to a radius of $1 \mathrm{~mm}$ ) and low financial cost.

The $\mathrm{CO}_{2}$ laser was also used hand-held in nine operations. It appeared to be very effective in excising large relatively superficial neoplasms such as meningiomas or gliomas.

CHANGES IN REGIONAL CEREBRAL BLOOD FLOW, CEREBRAL CONDUCTION TIME, AUTOREGULATION AND REACTIVITY FOLLOWING EXPERIMENTAL SUBARACHNOID HAEMORRHAGE

J Jakubowski, BA Bell, L Symon, M Zawirski (London)

In order to study the cerebral circulatory and neurophysiological effects of subarachnoid haemorrhage, the posterior communicating artery was divided in a series of baboons. Using implanted electrodes and hydrogen clearance, rCBF autoregulation and reactivity were measured for up to 3 months. Central conduction time was also studied by computer averaging of somatosensory evoked po tentials. Five animals were studied, threec with only mild sequelae (grade 1 and 2)? and two with more severe sequelae (grade 3 and 4). In the former, after the haemorrhage, there was immediate reduction of cerebral blood flow in all areas which was followed by recovery to values higher than control; increased flows were also seen two days later. By contrast, in the more severely affected animals, the blood flow remained low. Irrespective of the clinical grading, autoregulation in the cerebral cortex was impaired diffusely in all five animals but showed greater improvement in animals in grade 1 and 2 . On the other hand, reactivity of the cerebral circulation to changes in $\mathrm{PaCO}_{2}$ was depressed in only the most severely affected animals in regions corresponding to clinical deficits. Neural conduction within the nervous system was delayed in the latter group also.

The authors suggested that their observations might be clinically relevant : (1) high cerebral blood flows after subarachnoid haemorrhage might be favourable prognostically; (2) prolonged central conduction time might indicate ischaemia; (3) arterial hypertension and 
control ventilation might be justified in some patients following aneurysm surgery.

INTRACRANIAL LESION LOCALISATION: A SIMPLE METHOD USING CT SCANNING

\section{NI Azzam (Sheffield)}

Bony landmarks towards the base of the skull and the ventricular system at a higher level, help to localise intracranial lesions on CT. In their absence, the localisation of small lesions, especially high convexity ones is difficult. The difficulties are compounded by varying degrees of neck flexion of the scanned patient. This paper described a marking system which could be used to overcome some of these difficulties.

The method started by applying a special circumferential bandage to the patient's head with its edge parallel to the light beam emitted from the scanner gantry. When the lesion had been demonstrated on the scan, the same cut was reproduced after placing a radio-opaque marker on the bandage over the site of the lesion. Another bandage with bariumsulphate monofilaments sewn on its material in a grid fashion was applied to the head and a lateral skull radiograph taken. The information learnt from the skull radiograph could be transferred to the patient's scalp with relative ease.

Other previous methods of localisation were considered to be insufficiently accurate and to be complicated and time consuming. Computer-assisted systems although highly effective require the help of an expert radiologist to perform and the information gained can only be transferred to the patient using a number of fairly complicated measurements. The method prescribed was recommended as being simple, practical and not time consuming. Construction of the bandage was easy and cheap.

ENOLASE STUDIES IN THE CEREBRO-SPINAL FLUID AND IN TUMOURS OF THE HUMAN NERVOUS SYSTEM

WR Timperley, JA Royds, CB Taylor, MA Parsons (Sheffield)

A sensitive biochemical marker of neuronal damage would have many applications in clinical practice and in research. The authors reviewed studies of the enzyme enolase that suggested its potential value.

CSF had been obtained from 28 patients with brain tumours and 41 controls. The total enolase activity was significantly higher in the former group when compared with total protein, creatine kinase, pyruv- ate kinase, lactate dehydrogenase and aldolase; enolase was the only enzyme that showed raised activity in the CSF of patients with low grade astrocytomas. The cellular localisation of enolase isoenzymes had been studied in tumours and in the normal human nervous system, using an immunoperoxidase PAP technique. In the normal nervous system, $\gamma$ enolase was found in neurones but not glia. By contrast, $a$ enolase was found in astrocytes, ependyma, capillary endothelial, Schwann cells and arachnoid and weak activity was seen in oligodendroglial. $a$ enolase was not seen in normal neurones and neither neurones nor glial stained for beta enolase. In the tumours, only $a$ enolase activity was found. This was demonstrated in 11 astrocytomas, three ependymomas, one mixed glioma and four meningiomas. Activity was weak or absent in three medulloblastomas, two neuroblastomas, four acoustic neuromas, and three oligodendrogliomas. Reactive cells from around the tumour stained strongly for $a$ enolase but necrotic and ischaemic cell showed loss of activity.

In discussion the correspondence between the cell type of origin and tumour activity was noted and the use of enolase as a marker for cell damage was discussed.

THE USEFULNESS OF SELECTIVE SACRAL NEURECTOMY IN NEUROGENIC BLADDER DYSFUNCTION

DMC Forster, S Clarke, N Philp, DG Thomas (Sheffield)

In the last decade improved case selection, by means of more sophisticated urodynamic studies, coupled with reduced surgical risks, have given impetus to the performance of selective section of sacral nerves. Doubts still remain about the risk of relapse and the long term benefits gained. The authors reported 18 patients on whom they had performed selective sacral neurectomy and who had been followed for a year or more. The patients included enuretics and paraplegics unable to use an indwelling catheter without leakage or rejection.

The immediate effects of the operation become modified with the passage of time, reflecting the recuperative or adaptive powers of the nervous system. On the other hand, denervation appeared to increase the sensitivity of some terminals to drugs administered to the patient. Lasting benefits from the operation have been found in 14 out of 18 patients who had been followed for a mean period of $2.9 \mathrm{yr}$. In some patients operation alone had been beneficial but in others benefit had resulted from the combination of operation and antidiuretic or anticholinergic medication, even though the latter had been ineffective before operation.

The authors suggested that criteria for performing the operation, in addition to failure of medical treatment, should include bladder over-distension, uninhibited detrusor activity, a distensible bladder without too small a functional capacity, and the ability to initiate micturition voluntarily, unless a catheter regimen of some form was envisaged. They also cautioned that care should be exercised in advising operations in patients with upper urinary tract dilatation, marked dyssynergia or psychiatric problems.

AUDITING THE PROCESS OF REFERRAL OF A COMMON NEUROSURGICAL EMERGENCY

RS Maurice-Williams, PL Richardson (London)

In order to assess the efficacy of the organisation of a regional neurosurgical service 76 consecutive cases of spinal cord compression were studied prospectively. The whole process by which each patient passed through the chain of medical referral was examined in depth Not only were the patients and their? relatives interviewed but the records of the referring sources were scrutinised and, where appropriate, the referring doctors were questioned by telephone.

Remarkable delays in diagnosis and treatment were commonplace. The mean interval from the first symptom of cord compression until reaching the neurosurgical unit was 104 days. During the referral process, the proportion of patients with minimal functional disability fell from $68 \%$ to $10 \%$. In 48 cases, clearly avoidable delays had occurred. A wide range of reasons for these could be ascertained. Under the care of the family doctor, diagnostic error was a common problem, while at the referring hospital a lack or any sense of urgency was often present.

The effect of these delays on final outcome was surprisingly small by comparison with other factors in the natural history of the disease process. Only three patients seemed to have suffered permanent neurological disability as a result of lack of efficiency of the health care system. 
A METHOD FOR PRE-OPERATIVE LOCALISATION OF INTRACRANIAL PATHOLOGY USING THE ISOTOPE SCAN

MP Powell, DT Hope, PA Smith, HB Coakham (Bristol)

Localisation of intracranial tumours by CT scan alone can be particularly difficult if the lesion is small or peripherally placed. A method for accurately localising intracranial tumours, by a modification of isotope scanning was presented.

A $20 \mathrm{mCi}$ dose of Tc 99 labelled glucoheptonate was given intravenously $90 \mathrm{~min}$ prior to the scan. The patients were positioned in the true lateral position under the scannning head of the gamma camera and an image obtained on the persistence oscilloscope. The tumour showed as a white spot. A $100 \mu \mathrm{Ci}$ cobalt point source was then brought in from the periphery to touch the scalp, followed by the persistence oscilloscope until it overlay the tumour "blush". A skin mark was made at this point and a $1 \mathrm{~cm} \times 3 \mathrm{~mm}$ lead disc taped over it. A normal isotope scan image was then accumulated, checking the position of the mark relative to the tumour. The mark was then used to centre a craniotomy or a burr hole. If needed a vertex image was also made in the same way, and the two skin marks used as vectors when approaching the lesion.

The method had been used in 25 patients and proved accurate in each. The authors considered that its simplicity and reliability represented an advance over other complex methods that depended upon the CT scan.

COLLAGENOZYTIC ACTIVITY OF BRAIN TUMOURS IN-VITRO

AN Halaka, RM Gibson, CC Bird, RAD Bunning, JJ Reynolds (Leeds and Cambridge)

Breakdown of extracellular matrix by enzymes is thought to facilitate tumour invasion. Since collagen is the principal structural protein in fibroconnective and osseous tissues, the process of collagenolysis has been the subject of recent interest. Collagenase is usually found in a latent form and it has been suggested that this is an enzyme-inhibitor complex. Collagen breakdown could be produced either by increasing the production of active collagenase or decreasing the inhibitor.

The authors had studied the production of collagenase and collagenase inhibitor by 54 brain tumoùrs ( 29 meningiomas, nine metastases, six gliomas; five pituitary adenomas and five others) in short 'term tissue culture. Collagenase was assayed by measuring the release of (14c) labelled peptides from thermally reconstituted trypsin-resistant fibrils of acetylated rat skin collagen. Collagenase inhibitor was assayed against active rabbit bone collagenase of known strength. Meningiomas produced negligible levels of collagenase. Two metastatic tumours produced high levels of the enzyme. Dura from a patient with malignant meningioma and dura cultured with glioblastomas produced significant levels of collagenase. Almost all tumours produced a collagenase inhibitor. Invasive tumours produced less inhibitor than non-invasive tumours. Production of the inhibitor could be abolished by freezing or thawing tumour tissue before culture of by addition of cycloheximide to culture medium showing that the inhibitor is synthesised by the tumour. Collagenase inhibitor was also found in the media from cultured dura.

It was postulated that the host tissues may be stimulated by the tumour to produce collagenase, and thus facilitate tumour invasion.

PRELIMINARY COMMUNICATION-VOLUME MEASUREMENT OF INTRACRANIAL LESIONS BY CT

NA Lewtas (Sheffield)

The introduction of the Diagnostic Enhancement Package by EMI Limited has, on their equipment, made volume measurement a relatively quick and simple calculation. With this package, the perimeter of lesions can be traced with a light pen, and the number of pixels in this area is immediately calculated by the computer. This process is repeated in sequential slices traversing the whole of the lesion. The volume is then calculated as the product of the slice thickness and the sum of the pixel areas in the relevant sections.

The diagnostic package readily calculates the mean densities of lesions, and of the surrounding normal brain. The border of a lesion is derived by taking the mean of the density of the lesion and the density of the surrounding brain. This is traced at a narrow window width of 20 Hounsfield units.

The author had conducted phantom experiments and obtained an accuracy of volume estimation of over $90 \%$. Only minor discrepancies were found in comparing these estimations with displacement volumes of well defined resected tumours, such as meningioma. There were two main reasons for discrepancies: incomplete removal of the tumour, and shrinkage of the specimen after resection due to blood loss following occlusion of the feeding vessels. The method was suggested to be of possible value in the management of intracerebral clots, abscesses, and the response of some lesions to nonsurgical treatment.

CALCIUM IONS AND CALCIUM ANTAGONISTS IN CEREBRAL ISCHAEMIA

RJ Harris, L Symon, NM Branston (London)

The authors had investigated the effect of a calcium antagonist, nimodipine, on ischaemic pathophysiology, in an attempt to reduce the intracellular accumulation of calcium in ischaemia. This has been associated with the pathological changes that lead to cell death. They employed an acute primate model of cerebral ischaemia produced by occlusion of the middle cerebral artery under alpha-chloralose anaesthesia. Nimodipine was administered by continuous carotid arterial infusion at $0.6 \mu \mathrm{g} / \mathrm{kg} / \mathrm{min}$. During the experiment blood flow and extracellular potassium $\left(\mathrm{K}_{\mathrm{e}}\right)$ and calcium $(\mathrm{Ca})$ activities were measured using microelectrodes placed in the same brain region and, at the end of the experiment, local brain water content was measured by a specific gravity method.

Nimodipine infusion was found to increase blood flow by $25 \%$ and to decrease $\mathrm{CO}_{2}$ reactivity by $75 \%$. The drug did not affect autoregulation to increased blood pressure, but did drastically impair regulation to reduce blood pressure. Nimodipine increased by almost two-fold the residual flow after middle cerebral artery occlusion. In untreated animals the blood flow threshold for disruption of ion homeostasis was found at a flow of around 10 $\mathrm{ml} / 100 \mathrm{~g} / \mathrm{min}$. $\mathrm{K}_{\mathrm{e}}$ was shown to rise first and to reach about $13 \mathrm{mM}$ before $\mathrm{Ca}_{\mathrm{e}}$ began to fall. The blood flow threshold at which ion homeostasis was disrupted was higher in animals treated with nimodipine, but the relationship between the $\mathrm{K}_{e}$ and the $\mathrm{Ca}_{e}$ change was not altered. The accumulation of oedema over a 90 min occlusion period was increased in treated animals. This increase was seen irrespective of the degree of ischaemia that had been present in the tissue. Nimodipine did not stop influx of calcium into the cells.

The data showed that nimodipine $N$ redistributed flow after arterial occlusion and might be expected to reduce the 
amount of brain that was ischaemic. On the other hand, any area where flow was reduced might, in practice, be rendered more susceptible to ischaemic damage.

EFFECT OF PROSTACYCLINE AND DYPRIDAMOLE IN PLATELET AND FIBRIN AGGREGATION IN MICROARTERIAL ANASTAMOSIS IN RATS

A scanning electron microscope study

UM Chowdhary (Dublin)

Microvascular anastamosis in small vessels is limited by the occurrence of thrombosis at the operative site. The author had devised an experimental model to study platelet and fibrin aggregation in microarterial end-to-end anastamosis and their modification by various anti-platelet anti-thrombotic agents. The common carotid arteries of Wistar rats were anastamosed using 75 micron needles and 10-0 Polyamide sutures. Three groups were studied. 1, Control; 2, Prostacycline treated animals and 3, Dypridamole treated animals. Ten microarterial anastamoses were made in each group. The vessels were fixed in vivo with gluteraldehyde. Quantitative and qualitative assessments of the scanning electron microscopic findings were made.

Both Prostacycline and Dypridamole reduced platelet and red blood cell aggregation at and near the suture site. $68 \%$ of the endothelial surface in the control group was covered with platelet, fibrin and red blood cells. By comparison, the areas covered were $15 \%$ and $18 \%$ respectively in animals treated with Prostacycline or Dypridamole. Fibrin deposition was not prevented by treatment but platelets and red blood cells did not adhere to the fibrinous strands.

The author suggested that the use of these drugs might improve the patency rate of microarterial anastamosis in arteries as small as $0.75 \mathrm{~mm}$ and thereby extend the scope of microvascular surgery.

AN UNUSUAL CASE OF "JUVENILE SUBDURAL HYGROMA"

NT Gurusinghe, AE Richardson (London) The authors described a 35-year-old mentally subnormal man who had presented with a 3 year history of progressive right hemiparesis and increasedspeech impairment. His investigation showed bilateral communicating subdural collections, with extensive calcification of the visceral membrane. In addition there was a large left sided arachnoid cyst. The subdural space proved to contain thick pus which grew Enterobacter clocae. After repeated aspirations, eventually bilateral craniotomy was performed; the calcified membrane and bone flaps were removed and the subdural space drained. Despite antibiotic treatment the patient was left mute and totally dependent.

In discussion the relationship of subdural hygromas to middle cranial fossa arachnoid cyst was emphasised; the rarity both of calcification of the membranes and of infection of a preexisting subdural haematoma were stressed; and the management difficulties were noted.

MICROADENOMECTOMY AND POSTOPERATIVE FOLLOW-UP IN CUSHING'S DISEASE R Fahlbusch, OA Muller (Munich)

Pituitary dependent Cushing's disease is uncommon and methods of management vary. The authors had treated an unusually large series by transsphenoidal operation and reported their results. Fifty four patients had undergone operation and a microadenoma was found in 50 . In one of the remaining patients a total hypophysectomy was performed; the other patients were treated by adrenalectomy. Forty-five of the 50 patients with a microadenoma had been followed for one year or more. In 31 patients operation was followed by a remission of clinical and biochemical abnormalities. Measurements of ACTH showed that most of these patients had transient pituitary insufficiency after operation but permanent hormone replacement was not necessary. The disease occurred in three

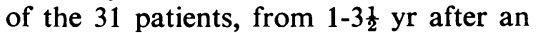
apparently successful operation. In the 14 patients who did not remit, ACTH levels remained high, indicating an operative failure. These patients had further treatment, either by total hypophysectomy or bilateral adrenalectomy. Immunohistochemical studies of the tumour and surround tissue were performed by Drs Fehm, Voigt and Martin but the findings did not correlate with the outcome of operation.

The authors concluded that transsphenoidal selective microacenomectomy should be the first step in the treatment of Cushing's Disease because it relieved the majority of patients of hypercortisolism without incurring endocrine deficiency. On the other hand the occurrence of failures or recurrence should be recognised, possibly reflecting a hypothalamic component in the aetiology of the disease. 